\title{
1 Conserved anti-inflammatory effects and sensing of butyrate in zebrafish
}

2 Pradeep Manuneedhi Cholan ${ }^{1}$, Alvin Han ${ }^{2}$, Brad R Woodie ${ }^{1,2}$, Angela RM Kurz ${ }^{3}$, Warwick

$3 \mathrm{~J}_{\text {Britton }}{ }^{1,4,5}$, Lihua $\mathrm{Ye}^{2}$, Zachary C Holmes ${ }^{2}$, Jessica R McCann ${ }^{2}$, Lawrence A David ${ }^{2}$,

4 John F Rawls ${ }^{2}$, Stefan H Oehlers ${ }^{1,4}$

$5{ }^{1}$ Tuberculosis Research Program at the Centenary Institute, The University of Sydney,

6 Camperdown NSW 2050, Australia

$7{ }^{2}$ Department of Molecular Genetics and Microbiology, Duke Microbiome Center, Duke

8 University School of Medicine, Durham NC 27710, USA

$9{ }^{3}$ Centenary Imaging and Sydney Cytometry at the Centenary Institute, The University of

10 Sydney, Camperdown NSW 2050, Australia

$11^{4}$ The University of Sydney, Discipline of Infectious Diseases and Immunology, Faculty of

12 Medicine and Health, and Marie Bashir Institute, Camperdown NSW 2050, Australia

$13{ }^{5}$ Department of Clinical Immunology, Royal Prince Alfred Hospital, Camperdown, NSW

142050 Australia

15

16 Corresponding author: Dr Stefan Oehlers stefan.oehlers@sydney.edu.au

17 ORCID: 0000-0003-0260-672X Twitter: @ oehlerslab

\section{Abstract}

20 Short chain fatty acids (SCFAs) are produced by microbial fermentation of dietary fiber in the 21 gut. Butyrate is a particularly important SCFA with anti-inflammatory properties and is 22 generally present at lower levels in inflammatory diseases associated with gut microbiota 23 dysbiosis in mammals. We aimed to determine if SCFAs are produced by the zebrafish 24 microbiome and if SCFAs exert conserved effects on zebrafish immunity as an example of the 25 non-mammalian vertebrate immune system. We demonstrate that bacterial communities from 
26 adult zebrafish intestines synthesize all three main SCFA in vitro, although SCFA were below

27 our detectable limits in zebrafish intestines in vivo. Immersion in butyrate, but not acetate or

28 propionate, reduced the recruitment of neutrophils and M1-type pro-inflammatory

29 macrophages to wounds. We found conservation of butyrate sensing by neutrophils via

30 orthologs of the hydroxycarboxylic acid receptor 1 (hcarl) gene. Neutrophils from Hcar1-

31 depeleted embryos were no longer responsive to the anti-inflammatory effects of butyrate,

32 while macrophage sensitivity to butyrate was independent of Hcar1. Our data demonstrate

33 conservation of anti-inflammatory butyrate effects and identify the presence of a conserved

34 molecular receptor in fish.

35 Keywords: zebrafish, butyrate, short chain fatty acid, inflammation, neutrophil, macrophage,

36 tumor necrosis factor

\section{Introduction}

Short chain fatty acids (SCFAs) are microbial metabolites produced in the gut by the anaerobic fermentation of dietary fiber and protein in the large intestine ${ }^{1}$. The most abundant SCFAs are acetate, butyrate, and propionate. In addition to providing the host with an energy source, microbially-derived SCFAs exert anti-inflammatory effects through inhibition of histone deacetylates (HDAC) and activation of G-protein coupled receptors (GPCRs) ${ }^{2}$. Most research on SCFAs has reported their anti-inflammatory properties in mammals ${ }^{3-5}$. However, the anti-inflammatory mechanism responsible for the anti-inflammatory effects of SCFA administration has not been reported in fish species to date.

Zebrafish are an important model of vertebrate gut physiology with key experimental advantages including high fecundity, transparency, and well-developed gut digestive function 
51 across vertebrates, including the sensitivity of zebrafish intestinal epithelial cell progenitors to

52 butyrate ${ }^{7-9}$. However, SCFA production has not been previously observed in the intestines of

53 zebrafish, and it is unclear if the intestinal lumen of the zebrafish intestine provides a suitable

54 niche for SCFA production ${ }^{9}$. Nevertheless, detectable amounts of acetate, butyrate, and

55 propionate have been measured in several species of teleosts ${ }^{10-14}$.

57 We have used conservation between mammalian intestinal function and immunity to create 58 zebrafish models of human intestinal inflammation ${ }^{15,16}$. Key pattern recognition molecule 59 families, such as the Toll-like receptors and Nod-like receptors, are evolutionarily ancient, 60 and have conserved roles in zebrafish intestinal immunity ${ }^{7,8,17,18}$. However, conservation of 61 host molecules responsible for sensing SCFAs has not been explored in teleosts. Mammals 62 utilize a wide range of molecules to sense SCFAs including G protein-coupled receptors 63 (GPRs) GPR81 (also known as HCAR1) which is primarily present on immune cells and 64 GPR109A which is expressed on intestinal epithelial cells. Microbially-derived SCFAs also 65 exert direct effects on host physiology through histone deacetylase (HDAC) inhibition ${ }^{19-21}$.

67 In this study, we investigated whether SCFAs are produced in the zebrafish intestine, and if 68 the anti-inflammatory effects and sensing of SCFAs are conserved in zebrafish. We find that 69 the pattern of SCFA production by the zebrafish intestinal microbiota is different from that 70 seen in mammals, but that the anti-inflammatory effects mechanisms of butyrate are 71 conserved across vertebrate species and development regardless of the ability of their 72 endogenous microbiota to produce measurable butyrate.

\section{Methods}

\subsection{Zebrafish handling}


76 Adult zebrafish were housed at the Centenary Institute (Sydney Local Health District AEC

77 Approval 17-036) and Duke University School of Medicine ((Duke Institutional Animal Care

78

79

80

81

82

83

84

85

86

87

88 and Use Committee Protocol Approval A115-16-05). Adult zebrafish experimentation was approved by the Institutional Animal Care and Use Committees of Duke University approval A115-16-05. Zebrafish adults were reared, housed, and fed as previously described ${ }^{22}$. All zebrafish embryo research experiments and procedures were completed in accordance with Sydney Local Health District animal ethics guidelines under approval 17-036. Zebrafish embryos were obtained by natural spawning and embryos were maintained and raised in E3 media at $28^{\circ} \mathrm{C}$.

\subsection{SCFA quantification from adult zebrafish}

Adult zebrafish were euthanized with $200-300 \mathrm{mg} / \mathrm{L}$ of ethyl 3-aminobenzoate methanesulfonate (tricaine) (Sigma, E10521) prior to dissection. For each sample, intestines dissected from five adult $(90+\mathrm{dpf})$ EK WT zebrafish males (roughly $0.2 \mathrm{~g}$ total) were pooled and homogenized using a Precellys 24 High-Powered Bead Homogenizer at $5500 \mathrm{rpm}$ for 3 cycles at 20 seconds per cycle with a 10 second delay between cycles. Samples were then acidified with $\mathrm{HCl}$ to a $\mathrm{pH}$ below 3, pelleted by centrifugation, and the supernatant was harvested. Filtered supernatant was stored at $-80^{\circ} \mathrm{C}$ until quantification.

SCFA quantification as carried out on an Agilent 7890B GC FID, with an HP-FFAP capillary column ( $25 \mathrm{~m}$ length, ID $0.2 \mathrm{~mm}$, film thickness $0.33 \mu \mathrm{m})$. Concentrations were determined using a linear model fit of a standard curve that encompasses the sample concentration range. Standardized concentrations used for each C2-C5 SCFA were as follows: 0.2, 0.5, 1, 2, 4, and $8 \mathrm{mM}$. 


\subsection{In vitro synthesis of SCFA by zebrafish gut commensals}

102 Freshly dissected intestines from four adult (6-month-old) EK WT zebrafish males and frozen

103 mouse fecal pellets were homogenized under reducing conditions to preserve the anaerobes.

104 Samples were handled in a Coy anaerobic chamber and used to inoculate tubes containing

105 brain-heart infusion (BHI) media (Thermo Scientific, OXOID) or Gifu anaerobic media

106 (Sigma), both supplemented with deoxygenated hemin and vitamin $\mathrm{K}$ to a final concentration

107 of $12.5 \mathrm{mg} / \mathrm{L}$ of hemin and $2.5 \mathrm{mg} / \mathrm{L}$ of vitamin $\mathrm{K}$.

108

109 Tubes were incubated in a sealed anaerobic jar with a Gas-Pak (Becton and Dickinson) to

110 maintain anaerobic conditions at $28^{\circ} \mathrm{C}$ for 24 hours. Samples were then acidified to a $\mathrm{pH}$

111 below 3 with $\mathrm{HCl}$, pelleted by centrifugation, and the supernatant was harvested. Filtered

112 supernatant was stored at $-80^{\circ} \mathrm{C}$ until quantification with methods identical to those listed

113 above in Section 2.2.

114

\section{$115 \quad 2.4$ Drug treatments}

116 Embryos were treated with $30 \mathrm{mM}$ sodium acetate (Sigma; S2889), $30 \mathrm{mM}$ sodium butyrate

117 (Sigma; B5887), $30 \mathrm{mM}$ sodium propionate (Sigma; P1880), $50 \mu \mathrm{g} / \mathrm{mL}$ dexamethasone

118 (Sigma; D4902), or $100 \mathrm{mM}$ 6-aminocaproic acid (Sigma; A2504). Drug stocks were

119 dissolved in DMSO or PBS and added to E3.

\subsection{Tail wounding experiment}

122 Caudal fin amputation was performed on 5 dpf embryos unless otherwise indicated. Zebrafish

123 embryos were anesthetized with tricaine. Embryos were cut posterior to the notochord using a

124 sterile scalpel. Embryos were then recovered to fresh $\mathrm{E} 3$ and kept at $28^{\circ} \mathrm{C}$. 


\subsection{Imaging}

127 Live zebrafish embryos were anesthetized using tricaine, mounted on $3 \%$ methylcellulose

128 (Sigma, M0512), and imaged using a Leica M205FA. ImageJ software was used to quantify

129 the fluorescent pixel count within $100 \mu \mathrm{m}$ of the wound site.

130

131 Additional high resolution and time-lapse microscopy was carried out on anesthetized

132 embryos embedded in $1 \%$ low melt agarose in a 96 well-plate with a Leica SP8 confocal

133 microscope or Deltavision Elite microscope.

\subsection{Neutrophil tracking}

136 Time-lapse images were processed and analyzed using ImageJ. Neutrophils were tracked

137 using the Trackmate plugin in ImageJ software and further quantified using Chemokine and

138 Migration tool software (Ibidi).

\subsection{Germ-free derivation and microdissection of embryos}

141 Germ-free zebrafish were created and maintained as previously described ${ }^{23}$. The gut and

142 body of $5 \mathrm{dpf}$ embryos were separated using a 25-gauge needle and added to Trizol LS

143 (Invitrogen; 10296010) for RNA extraction.

145 2.9 RNA extraction, cDNA synthesis and quantitative PCR (qPCR)

14610 - 20 zebrafish embryos were pooled and lysed using a 25-gauge needle in Trizol LS for

147 RNA extraction. cDNA was synthesized using a High-capacity reverse transcription kit

148 (ThermoFisher Scientific, 4368814). qPCR was carried out using Power UP SYBR green

149 master mix (ThermoFisher Scientific, 4385610) on a CFX96 Real-Time system (BioRad).

150 Primer pairs (5`- 3`); $18 s \quad$ TCGCTAGTTGGCATCGTTTATG and 
151 CGGAGGTTCGAGACGATCA; harl CATCGTCATCTACTGCTCCAC and

152 GCTAACACAAACCGCACA.

153

$154 \quad 2.10$ gRNA synthesis and CRISPR injections

155 gRNA templates for hcarl-2 $\quad\left(5^{`}-3^{\prime}\right)$ : $\quad$ Target 1

156 TAATACGACTCACTATAGGTACCGGCGGCTCGATTGGGTTTTAGAGCTAGAAATA

157 GC, $\quad$ Target 2

158 TAATACGACTCACTATAGGAGCAACTCTCGCTTCACTGTTTTAGAGCTAGAAATA

159 GC $\quad$, Target 3

160 TAATACGACTCACTATAGGGATTCGAGAGATGTTACTGTTTTAGAGCTAGAAATA

161 GC. gRNA was synthesized as previously described ${ }^{24}$.

162

163 A 1:1 solution of gRNA and $500 \mu \mathrm{g} / \mathrm{mL}$ of Cas9 nuclease V3 (Integrated DNA Technology)

164 was prepared with phenol red dye (Sigma, P0290). Freshly laid eggs were collected from

165 breeding tanks and the solution was injected in the yolk sac of the egg before the emergence

166 of the first cell with a FemtoJet 4i (Eppendorf).

167

$168 \quad 2.11$ Statistics

169 All statistical analyses (t-tests and ANOVA where appropriate) were performed using

170 GraphPad Prism8. Outliers were removed using ROUT, with $\mathrm{Q}=1 \%$. All data are

171 representative of at least 2 biological replicates.

172

173 Results

$174 \quad 3.1$ Zebrafish gut commensals are capable of producing SCFA ex vivo 
175 We initially tried to detect SCFAs in whole intestines and their contents dissected from

176 conventionally-reared adult zebrafish using gas chromatography but levels of acetate,

177 butyrate, or propionate were below our limit of detection of 0.00132 mmol SCFA per g of

178 tissue.

180 We next sought to determine if the conventional zebrafish gut microbiota had the capacity to

181 produce SCFAs using ex vivo culture on two rich medias, BHI and Gifu. We found that

182 microbial communities cultured from conventionally-reared adult zebrafish intestines were

183 able to synthesize acetate under both aerobic and anaerobic conditions (Figure 1). Butyrate

184 and propionate were only detected under anaerobic conditions. The highest concentrations of

185 SCFA were detected under anaerobic conditions in BHI where acetate, propionate, and

186 butyrate were present in a roughly 90:5:5 ratio.

\subsection{Butyrate reduces the recruitment of zebrafish neutrophils to a wound}

189 We first observed the effect of SCFAs (acetate, butyrate and propionate) on neutrophil

190 migration following a tail wound injury using $\operatorname{Tg}(\operatorname{lyz} C: D s \operatorname{Red})^{n z 50}$ and $\operatorname{Tg}(\operatorname{lyz} C: G F P)^{n z 117}$

191 transgenic zebrafish lines where neutrophils are fluorescently labelled (Figure 2A). We

192 observed a significant reduction in the number of recruited neutrophils at 6 hours post

193 wounding (hpw) in embryos exposed to butyrate by immersion (Figure 2B). There were no

194 changes seen with acetate or propionate, but dexamethasone, a corticosteroid anti-

195 inflammatory used as a positive control, reduced neutrophil recruitment as expected.

197 We next assessed the quality of neutrophil recruitment by intravital imaging. We observed

198 reduced neutrophil velocity (Figure 2C), and increased meandering index (total distance

199 traveled / Euclidean distance) in butyrate-treated embryos (Figure 2D). 
201 We next sought to determine if butyrate sensitivity is dependent on intestinal maturity by

202 repeating the tail wound experiment using 2 dpf embryos, a developmental stage prior to

203 significant intestinal morphogenesis ${ }^{25}$, and found neutrophil recruitment was overall reduced

204 compared to $5 \mathrm{dpf}$, but still further inhibited by butyrate immersion (Figure 2E).

205

\subsection{Butyrate reduces the proportion of TNF positive macrophages at the wound site}

207 Next, we examined the effect of SCFAs on macrophage recruitment and polarization

208 following wounding. $\operatorname{Tg}(\text { mfap4:tdTomato })^{x t 12}$ transgenic zebrafish were used to visualize

209 macrophage numbers ${ }^{26}$. Relative to control embryos, macrophage recruitment was reduced

210 by butyrate and increased by propionate treatment at $6 \mathrm{hpw}$ (Figure 3A). Consistent with a

211 lack of effect on neutrophil recruitment, acetate treatment did not affect the number of

212 recruited macrophages, and as the positive control anti-inflammatory dexamethasone

213 significantly reduced macrophage recruitment. These changes were maintained at $24 \mathrm{hpw}$

214 when inflammation is in the resolution phase (Figure 3B).

215

216 We next used the $\operatorname{TgBAC}(\text { tnfa:gfp })^{\text {pd1026 }}$ line to monitor inflammatory gene expression ${ }^{27}$. As

217 expected, since macrophages are the primary producers of TNF in zebrafish embryos, total

218 TNF promotor fluorescence area at $24 \mathrm{hpw}$ reflected the trend of macrophages recruitment in

219 each treatment condition with butyrate, but not acetate or propionate, reducing TNF promoter

220 expression (Figure 3C). We next crossed the TgBAC(tnfa:gfp pdion and

$221 T g(\text { mfap4:tdTomato })^{x t 12}$ lines to monitor macrophage inflammatory polarization as defined by

222 inflammatory TNF promotor expression (Figure 3D) ${ }^{28}$. Butyrate treatment reduced the

223 percentage of $T g B A C(\text { tnfa: } g f p)^{p d 1026}$ positive macrophages (Figure 3E). 


\subsection{Butyrate does not have toxic effects as measured by hemostatic indices}

226 Butyrate has been previously shown to reduce the proliferation of zebrafish intestinal

227 epithelial cells ${ }^{9}$. This raises the possibility that inhibition of leukocyte recruitment by

228 butyrate immersion was due to toxicity. Changes to zebrafish hemostasis have been observed in models of toxicity and inflammation ${ }^{29,30}$.

231 We used Tg(fabplOa:fgb-EGFP $)^{\text {mit001 }}$, where fibrin clots are visualized by GFP deposition, 232 and $T g(-6.0 i t g a 2 b: e G F P)^{l a 2}$, where thrombocytes are GFP-labeled, transgenic zebrafish lines

233 to monitor hemostasis following transection of the dorsal aorta and posterior cardinal vein ${ }^{31}$,

$234{ }^{32}$. We stabilized clots with aminocaproic acid as a positive control ${ }^{33}$. Fibrinogen 235 accumulation in $T g(f a b p 10 a: f g b-E G F P)^{m i 4001}$ embryos was unchanged at the wound site in 236 response to butyrate treatment, however we noted that propionate treatment caused increased 237 fibrinogen accumulation (Supplementary Figure 1A). No changes were observed in 238 thrombocytes accumulation in the Tg(-6.0itga2b:eGFP $)^{l a 2}$ line following any of the SCFA 239 treatments (Supplementary Figure 1B).

\subsection{Characterization of the zebrafish hydrocarboxylic acid receptor 1}

242 Hydrocarboxylic acid receptor 1 (HCARl) is an important receptor for butyrate in mammals

$243{ }^{19}$. We identified a conserved region of zebrafish chromosome 10, human chromosome 12, 244 and mouse chromosome 5 containing density regulated re-initiation and release factor (denr), 245 coiled-coil domain-containing 62 (ccdc62), huntingtin interacting protein 1 related a (hip1ra)

246 loci and the single exon hcar family (Figure 4A). Two copies of the putative zebrafish hcarl 247 (with 93\% identity and lacking sufficient divergence to differentiate by PCR) were identified 248 as annotated by the single-entry NM_001163295.1 (Danio rerio hydroxycarboxylic acid 249 receptor 1-2 (hcar1-2), mRNA) suggesting the possibility of a tandem duplication event 
250 (Supplementary File). Kuei et al. previously annotated what we refer as hcarla as gpr81-2

251 and hcarlb as gpr81-1 ${ }^{34}$. The predicted 933/936 bp transcript of hcarla/hcarlb is expected

252 to give rise to a $310 / 311$ amino acid protein with $89 \%$ amino acid identity. The

253 Hcar1a/Hcar1b hypothetical proteins contain 7 predicted transmembrane helix domains

254 characteristic of a GPCR and an E-value of $7.05^{-157} / 3.21^{-166}$ for the HCAR subfamily ${ }^{35}$. The

255 predicted zebrafish proteins have approximately $43 \%$ identity to the human HCAR1, HCAR2,

256 and HCAR3; and mouse HCAR1 and HCAR2 proteins.

257

258 Mammalian HCARl is highly expressed by intestinal epithelial cells and neutrophils ${ }^{19}$.

259 Compared to other immune cells, hcarl expression is enriched in zebrafish neutrophils ${ }^{36,37}$.

260 We sought to characterize expression of hcarl in the intestines of zebrafish embryos using

261 microdissection of $5 \mathrm{dpf}$ embryos. We found increased hcarl expression in dissected guts

262 compared to the rest of the embryo by RT-qPCR analysis (Figure 4B). Interestingly, there

263 were no significant changes observed with the absence of microbial colonization in germ-free

264 embryos (Supplementary Figure 2).

265

3.6 The anti-inflammatory effects of butyrate on neutrophils, but not macrophages, are

268 To determine if butyrate acts through the Hcar1 receptor, we next used CRISPR/Cas9

269 technology to knockdown hcarl expression in zebrafish embryos. We utilized three target

270 sites in hcarla, two of which had strong homology to sequences in hcarlb (Supplementary

271 File). We confirmed $\sim 50 \%$ transcript depletion by RT-qPCR for a shared hcarla/hcarlb

272 sequence (Figure 4C). Embryo development was morphologically normal compared to

273 embryos injected with control scrambled guide RNA-Cas9 complex (Figure 4D). 
275 We performed tail wound injury on hcarl knockdown embryos. Knockdown of hcarl

276 abrogated the effect of butyrate treatment on neutrophil recruitment (Figure 4E). As expected

277 from the lack of expression on macrophages, hcarl knockdown did not affect the reduced

278 macrophage recruitment (Figure 4F) and TNF promotor expression (Figure 4G) induced by

279 butyrate immersion.

280

281 Discussion

282 This study shows for the first time that commensal microbiota residing in the zebrafish 283 intestine are capable of producing SCFAs. Experimentally, we demonstrate that the effects, 284 and sensing, of butyrate are conserved between zebrafish and mammals. Out of the three main

285 SCFAs, only the anti-inflammatory effect of butyrate was found to be conserved in zebrafish

286 embryos. We applied the commonly used tail wounding model to demonstrate an anti-

287 inflammatory effect of butyrate on zebrafish neutrophils and macrophages. Using Crispr-Cas9

288 targeted mutagenesis, we also identified conserved butyrate responsiveness of the zebrafish

289 Hcar1 receptor.

290

291 The anti-inflammatory effects of butyrate have been established in numerous in vivo and in

292 vitro studies of mammalian hosts but not in fish ${ }^{38}$. Our zebrafish tail wound model

293 demonstrates conservation of this property in a bony fish. Bony fish diverge from mammals

294 approximate 420 million years ago suggesting that the sensing of microbially-derived SCFAs

295 has been conserved from a common ancestor.

296

297 Our finding that immune cells are responsive to butyrate even before intestinal lumen

298 formation in early embryonic development is surprising as $2 \mathrm{dpf}$ embryos are usually

299 contained within relatively impervious chorions that prevent microbial colonization of the 
300

301

302

303

304

305

306

307

308

309

310

311

312

313

314

315

316

324

embryo. This suggests that the ability to sense xenobiotic SCFAs is programmed alongside the ability to sense more traditional microbially-associated molecular patterns via pattern recognition molecules.

The HCAR1/GPR81 butyrate receptor is expressed by many mammalian innate immune cells ${ }^{19}$. Expression in zebrafish is strongest in granulocytes ${ }^{36,37}$. Our knockdown experiments further demonstrate Hcar1 is necessary for the butyrate sensitivity of neutrophils but not macrophages. Thus, the butyrate-Hcar1-neutrophil behavior axis is evolutionarily ancient.

Human macrophages in the presence of butyrate have been shown to differentiate into an M2 phenotype which exhibits anti-microbial and tissue reparative properties ${ }^{39,40}$. These effects are independent of HCAR1 signal transduction and are believed to be an effect of butyrate acting as a histone deacetylase inhibitor ${ }^{41,42}$. SCFAs can permeate cell membranes through passive diffusion or through specific transporters such as the proton-coupled monocarboxylate-transporter 1 (MCT1) and sodium-coupled monocarboxylate-transporter 1 (SMCT1) ${ }^{43-46}$. Consistent with this literature, we show butyrate reduces the expression of pro-inflammatory TNF by zebrafish macrophages independent of Hcar1 expression. Our data suggests the HCAR1-independent immunosuppressive actions of butyrate may be conserved across vertebrate evolution.

Our data demonstrate that, under nutrient-rich in vitro conditions, gut commensal microbiota from adult zebrafish are capable of synthesizing the three most important SCFAs: acetate, propionate, and butyrate. However, the ratio of acetate, propionate, and butyrate produced under anaerobic conditions in BHI media (90:5:5) differed from the ratio typically observed in mammalian colons $(60: 20: 20)^{1}$. This may be due to the differing bacterial communities 
325 found in zebrafish and mammalian intestines. The most abundant bacterial phyla in the adult

326 zebrafish intestine are Proteobacteria and Fusobacteria, whereas mouse and human intestines

327 are dominated by members of phyla Bacteroidetes and Firmicutes ${ }^{47}$. Considering the SCFA

328 production we observed in vitro, our inability to detect SCFA in vivo was surprising. We

329 anticipate this could be due to rapid host or microbial metabolism of SCFA produced within

330 the zebrafish gut, or the composition of the diet fed to the zebrafish tested in this experiment.

331 Zebrafish are omnivores and were fed protein rich diets in the Duke aquaculture facility. A

332 diet with more SCFA substrates such as carbohydrates and fiber may yield detectable SCFA

333 production in situ.

334

335 Interestingly, we observed increased macrophage and fibrinogen clot accumulation at the

336 wound site following propionate treatment, indicative of a pro-inflammatory effect. Although

337 this is at odds with anti-inflammatory effects of propionate in mammals ${ }^{48-50}$, it is consistent

338 with evidence of an immunostimulatory effect of propionate in teleosts ${ }^{51,52}$.

340 Overall this manuscript provides further evidence of conserved mechanisms of host-microbe

341 interaction within vertebrates. We present evidence that immunological sensitivity to butyrate

342 is conserved across vertebrates. Furthermore, there is conservation of the molecular

343 machinery that senses butyrate even down to the responsiveness of individual leukocyte

344 lineages.

346 Acknowledgements

347 Funding: Australian National Health and Medical Research Council Project Grant

348 APP1099912; The University of Sydney Fellowship G197581; NSW Ministry of Health

349 under the NSW Health Early-Mid Career Fellowships Scheme H18/31086 to SHO. 
350 The authors acknowledge the facilities and the scientific and technical assistance of the

351 BioImaging Facility and Sydney Cytometry at Centenary Institute.

352 The authors declare no conflicts of interest.

\section{References}

355 1. den Besten G, van Eunen K, Groen AK, Venema K, Reijngoud DJ, Bakker BM. The role of short-chain fatty acids in the interplay between diet, gut microbiota, and host energy metabolism. J Lipid Res 2013; 54:2325-40.

2. Tan J, McKenzie C, Potamitis M, Thorburn AN, Mackay CR, Macia L. The role of short-chain fatty acids in health and disease. Adv Immunol 2014; 121:91-119.

3. Tedelind S, Westberg F, Kjerrulf M, Vidal A. Anti-inflammatory properties of the short-chain fatty acids acetate and propionate: a study with relevance to inflammatory bowel disease. World J Gastroenterol 2007; 13:2826-32.

363 4. Zheng N, Gao Y, Zhu W, Meng D, Walker WA. Short chain fatty acids produced by

364 colonizing intestinal commensal bacterial interaction with expressed breast milk are antiinflammatory in human immature enterocytes. PLoS One 2020; 15:e229283.

5. Baxter NT, Schmidt AW, Venkataraman A, Kim KS, Waldron C, Schmidt TM. Dynamics of Human Gut Microbiota and Short-Chain Fatty Acids in Response to Dietary Interventions with Three Fermentable Fibers. mBio 2019; 10.

6. Matty MA, Oehlers SH, Tobin DM. Live Imaging of Host-Pathogen Interactions in Zebrafish Larvae. Methods Mol Biol 2016; 1451:207-23.

7. Oehlers SH, Flores MV, Chen T, Hall CJ, Crosier KE, Crosier PS. Topographical distribution of antimicrobial genes in the zebrafish intestine. Dev Comp Immunol 2011; 35:385-91.

374 8. Oehlers SH, Flores MV, Hall CJ, Swift S, Crosier KE, Crosier PS. The inflammatory

375 bowel disease (IBD) susceptibility genes NOD1 and NOD2 have conserved anti-bacterial 376 roles in zebrafish. Dis Model Mech 2011; 4:832-41.

377 9. Kaiko GE, Ryu SH, Koues OI, Collins PL, Solnica-Krezel L, Pearce EJ, et al. The 378 Colonic Crypt Protects Stem Cells from Microbiota-Derived Metabolites. Cell 2016; 379 165:1708-20.

380 10. Romano N, Simon W, Ebrahimi M, Fadel AHI, Chong CM, Kamarudin MS. Dietary 381 sodium citrate improved oxidative stability in red hybrid tilapia (Oreochromis sp.) but 382 reduced growth, health status, intestinal short chain fatty acids and induced liver damage. 383 Aquaculture 2016; 458:170-6.

384 11. Titus E, Ahearn GA. Short-chain fatty acid transport in the intestine of a herbivorous 385 teleost. J Exp Biol 1988; 135:77-94.

386 12. Mountfort DO, Campbell J, Clements KD. Hindgut fermentation in three species of 387 marine herbivorous fish. Appl Environ Microbiol 2002; 68:1374-80.

388 13. Hao YT, Wu SG, Jakovlić I, Zou H, Li WX, Wang GT. Impacts of diet on hindgut 389 microbiota and short-chain fatty acids in grass carp (Ctenopharyngodon idellus). Aquaculture 390 Research 2017; 48:5595-605.

391 14. Ebrahimi M, Daeman NH, Chong CM, Karami A, Kumar V, Hoseinifar SH, et al. 392 Comparing the effects of different dietary organic acids on the growth, intestinal short-chain 393 fatty acids, and liver histopathology of red hybrid tilapia (Oreochromis sp.) and potential use 394 of these as preservatives. Fish Physiol Biochem 2017; 43:1195-207. 
15. Oehlers SH, Flores MV, Hall CJ, Wang L, Ko DC, Crosier KE, et al. A whole animal chemical screen approach to identify modifiers of intestinal neutrophilic inflammation. FEBS J 2017; 284:402-13.

16. Oehlers SH, Flores MV, Hall CJ, Crosier KE, Crosier PS. Retinoic acid suppresses intestinal mucus production and exacerbates experimental enterocolitis. Dis Model Mech 2012; 5:457-67.

17. Koch BEV, Yang S, Lamers G, Stougaard J, Spaink HP. Intestinal microbiome adjusts the innate immune setpoint during colonization through negative regulation of MyD88. Nat Commun 2018; 9:4099.

18. Cheesman SE, Neal JT, Mittge E, Seredick BM, Guillemin K. Epithelial cell proliferation in the developing zebrafish intestine is regulated by the Wnt pathway and microbial signaling via Myd88. Proc Natl Acad Sci U S A 2011; 108 Suppl 1:4570-7.

19. Ranganathan P, Shanmugam A, Swafford D, Suryawanshi A, Bhattacharjee P, Hussein MS, et al. GPR81, a Cell-Surface Receptor for Lactate, Regulates Intestinal Homeostasis and Protects Mice from Experimental Colitis. J Immunol 2018; 200:1781-9.

20. Arpaia N, Campbell C, Fan X, Dikiy S, van der Veeken J, deRoos P, et al. Metabolites produced by commensal bacteria promote peripheral regulatory $\mathrm{T}$-cell generation. Nature 2013; 504:451-5.

21. Furusawa Y, Obata Y, Fukuda S, Endo TA, Nakato G, Takahashi D, et al. Commensal microbe-derived butyrate induces the differentiation of colonic regulatory $\mathrm{T}$ cells. Nature 2013; 504:446-50.

22. Murdoch CC, Espenschied ST, Matty MA, Mueller O, Tobin DM, Rawls JF. Intestinal Serum amyloid A suppresses systemic neutrophil activation and bactericidal activity in response to microbiota colonization. PLoS Pathog 2019; 15:e1007381.

23. Melancon E, Gomez De La Torre Canny S, Sichel S, Kelly M, Wiles TJ, Rawls JF, et al. Best practices for germ-free derivation and gnotobiotic zebrafish husbandry. Methods Cell Biol 2017; 138:61-100.

24. Wu RS, Lam, II, Clay H, Duong DN, Deo RC, Coughlin SR. A Rapid Method for Directed Gene Knockout for Screening in G0 Zebrafish. Dev Cell 2018; 46:112-25 e4.

25. $\mathrm{Ng} \mathrm{AN,} \mathrm{de} \mathrm{Jong-Curtain} \mathrm{TA,} \mathrm{Mawdsley} \mathrm{DJ,} \mathrm{White} \mathrm{SJ,} \mathrm{Shin} \mathrm{J,} \mathrm{Appel} \mathrm{B,} \mathrm{et} \mathrm{al.}$ Formation of the digestive system in zebrafish: III. Intestinal epithelium morphogenesis. Dev Biol 2005; 286:114-35.

26. Walton EM, Cronan MR, Beerman RW, Tobin DM. The Macrophage-Specific Promoter mfap4 Allows Live, Long-Term Analysis of Macrophage Behavior during Mycobacterial Infection in Zebrafish. PLoS ONE 2015; 10:e0138949.

27. Marjoram L, Alvers A, Deerhake ME, Bagwell J, Mankiewicz J, Cocchiaro JL, et al. Epigenetic control of intestinal barrier function and inflammation in zebrafish. Proc Natl Acad Sci U S A 2015; 112:2770-5.

28. Nguyen-Chi M, Laplace-Builhe B, Travnickova J, Luz-Crawford P, Tejedor G, Phan QT, et al. Identification of polarized macrophage subsets in zebrafish. Elife 2015; 4:e07288.

29. McLeish JA, Chico TJ, Taylor HB, Tucker C, Donaldson K, Brown SB. Skin exposure to micro- and nano-particles can cause haemostasis in zebrafish larvae. Thromb Haemost 2010; 103:797-807.

30. Hortle E, Johnson KE, Johansen MD, Nguyen T, Shavit JA, Britton WJ, et al. Thrombocyte Inhibition Restores Protective Immunity to Mycobacterial Infection in Zebrafish. J Infect Dis 2019; 220:524-34.

31. Vo AH, Swaroop A, Liu Y, Norris ZG, Shavit JA. Loss of fibrinogen in zebrafish results in symptoms consistent with human hypofibrinogenemia. PLoS ONE 2013; 8:e74682.

32. Lin HF, Traver D, Zhu H, Dooley K, Paw BH, Zon LI, et al. Analysis of thrombocyte development in CD41-GFP transgenic zebrafish. Blood 2005; 106:3803-10. 
445

33. Liu Y, Kretz CA, Maeder ML, Richter CE, Tsao P, Vo AH, et al. Targeted mutagenesis of zebrafish antithrombin III triggers disseminated intravascular coagulation and thrombosis, revealing insight into function. Blood 2014; 124:142-50.

34. Kuei C, Yu J, Zhu J, Wu J, Zhang L, Shih A, et al. Study of GPR81, the lactate receptor, from distant species identifies residues and motifs critical for GPR81 functions. Mol Pharmacol 2011; 80:848-58.

35. Marchler-Bauer A, Bo Y, Han L, He J, Lanczycki CJ, Lu S, et al. CDD/SPARCLE: functional classification of proteins via subfamily domain architectures. Nucleic Acids Res 2017; 45:D200-D3.

36. Rougeot J, Torraca V, Zakrzewska A, Kanwal Z, Jansen HJ, Sommer F, et al. RNAseq Profiling of Leukocyte Populations in Zebrafish Larvae Reveals a cxcl11 Chemokine Gene as a Marker of Macrophage Polarization During Mycobacterial Infection. Frontiers in immunology 2019; 10:832.

37. Athanasiadis EI, Botthof JG, Andres H, Ferreira L, Lio P, Cvejic A. Single-cell RNAsequencing uncovers transcriptional states and fate decisions in haematopoiesis. Nat Commun 2017; 8:2045.

38. Inan MS, Rasoulpour RJ, Yin L, Hubbard AK, Rosenberg DW, Giardina C. The luminal short-chain fatty acid butyrate modulates NF-kappaB activity in a human colonic epithelial cell line. Gastroenterology 2000; 118:724-34.

39. Ji J, Shu D, Zheng M, Wang J, Luo C, Wang Y, et al. Microbial metabolite butyrate facilitates M2 macrophage polarization and function. Sci Rep 2016; 6:24838.

40. Schulthess J, Pandey S, Capitani M, Rue-Albrecht KC, Arnold I, Franchini F, et al. The Short Chain Fatty Acid Butyrate Imprints an Antimicrobial Program in Macrophages. Immunity 2019; 50:432-45 e7.

41. Davie JR. Inhibition of histone deacetylase activity by butyrate. J Nutr 2003; 133:2485S-93S.

42. Chang PV, Hao L, Offermanns S, Medzhitov R. The microbial metabolite butyrate regulates intestinal macrophage function via histone deacetylase inhibition. Proc Natl Acad Sci U S A 2014; 111:2247-52.

43. Miyauchi S, Gopal E, Fei YJ, Ganapathy V. Functional identification of SLC5A8, a tumor suppressor down-regulated in colon cancer, as a $\mathrm{Na}(+)$-coupled transporter for shortchain fatty acids. J Biol Chem 2004; 279:13293-6.

44. Ritzhaupt A, Wood IS, Ellis A, Hosie KB, Shirazi-Beechey SP. Identification and characterization of a monocarboxylate transporter (MCT1) in pig and human colon: its potential to transport L-lactate as well as butyrate. J Physiol 1998; 513 ( Pt 3):719-32.

45. Borthakur A, Priyamvada S, Kumar A, Natarajan AA, Gill RK, Alrefai WA, et al. A novel nutrient sensing mechanism underlies substrate-induced regulation of monocarboxylate transporter-1. Am J Physiol Gastrointest Liver Physiol 2012; 303:G1126-33.

46. Alarcon P, Manosalva C, Conejeros I, Carretta MD, Munoz-Caro T, Silva LMR, et al. d(-) Lactic Acid-Induced Adhesion of Bovine Neutrophils onto Endothelial Cells Is Dependent on Neutrophils Extracellular Traps Formation and CD11b Expression. Front Immunol 2017; 8:975.

47. Rawls JF, Mahowald MA, Ley RE, Gordon JI. Reciprocal gut microbiota transplants from zebrafish and mice to germ-free recipients reveal host habitat selection. Cell 2006; 127:423-33.

48. Provost KA, Smith M, Miller-Larsson A, Gudleski GD, Sethi S. Bacterial regulation of macrophage bacterial recognition receptors in COPD are differentially modified by budesonide and fluticasone propionate. PLoS One 2019; 14:e0207675. 

zebrafish.

49. Ciarlo E, Heinonen T, Herderschee J, Fenwick C, Mombelli M, Le Roy D, et al. Impact of the microbial derived short chain fatty acid propionate on host susceptibility to bacterial and fungal infections in vivo. Sci Rep 2016; 6:37944.

50. Park JW, Kim HY, Kim MG, Jeong S, Yun CH, Han SH. Short-chain Fatty Acids Inhibit Staphylococcal Lipoprotein-induced Nitric Oxide Production in Murine Macrophages. Immune Netw 2019; 19:e9. 51. Hoseinifar SH, Zoheiri F, Caipang CM. Dietary sodium propionate improved performance, mucosal and humoral immune responses in Caspian white fish (Rutilus frisii kutum) fry. Fish Shellfish Immunol 2016; 55:523-8.

52. Safari R, Hoseinifar SH, Kavandi M. Modulation of antioxidant defense and immune response in zebra fish (Danio rerio) using dietary sodium propionate. Fish Physiol Biochem 2016; 42:1733-9.

\section{Figure Legends}

\section{Figure 1: In vitro synthesis of SCFA by zebrafish gut microbiota}

Concentrations of short-chain fatty acids (SCFA) synthesized by conventional microbiota harvested from adult zebrafish intestines. SCFA content of nutrient media used to culture microbes is provided under "Negative". Asterisks indicate measurements within the range of standards. Error bars are shown as mean $\pm \mathrm{SE}, \mathrm{n}=2$. Bars without an asterisk indicate concentrations that were outside the standard range but were detectable.

\section{Figure 2: Butyrate reduces the recruitment of zebrafish neutrophils to a wound}

(A) Cartoon describing the standard cut site transecting the dorsal aorta and cardinal vein of a 5 dpf zebrafish embryo, and the fin cut site used for live imaging studies. (B) Neutrophil counts at 6 hpw. Each dot represents a single embryo. (C) Velocity of wound-recruited neutrophils calculated from live imaging studies. Each dot represents the average of 10 neutrophils from a single embryo. (C) Meandering index (Total distance/Euclidean distance) of wound-recruited neutrophils calculated from live imaging studies. Each dot represents the average of 10 neutrophils from a single embryo. (D) Neutrophil count at $6 \mathrm{hpw}$ in $2 \mathrm{dpf}$ 
524 Figure 3: Butyrate reduces macrophage recruitment to the wound site and pro-

525 inflammatory differentiation.

526 (A) Macrophage fluorescent area at $6 \mathrm{hpw}$. (B) Macrophage fluorescent area at $24 \mathrm{hpw}$. (C)

527 Total TNF promotor fluorescent area at the wound site after 24 hpw. (D) Representative

528 images of double transgenic red macrophage, green TNF promoter activity embryos tail

529 wounds at $24 \mathrm{hpw}$. Scale bar represents $100 \mu \mathrm{m}$. (D) Quantification of wound site TNF

530 expressing macrophages at $24 \mathrm{hpw}$.

531

532 Figure 4: Characterization of zebrafish hydrocarboxylic acid receptor 1 and responsiveness to butyrate.

534 (A) Synteny diagram illustrating HCARl in a conserved region of human chromosome 12,

535 mouse chromosome 5, and zebrafish chromosome 10. (B) Quantification of hcarl expression

536 in dissected gut and body of zebrafish embryos. Each dot represents a biological replicate of

537 at least 10 embryos. (C) Quantification of hcarl expression in 5 dpf embryos injected with

538 hcarl-targeting Crispr-Cas9 complexes. Each dot represents a biological replicate of at least

53910 embryos. (D) Morphology of the control and crispant embryos. Scale bar represents 100

$540 \mu \mathrm{m}$. (E) Quantification of neutrophil area at $6 \mathrm{hpw}$ in control and crispant embryos exposed to

541 butyrate by immersion. (E) Quantification of macrophage area at $6 \mathrm{hpw}$ in control and

542 crispant embryos exposed to butyrate at $6 \mathrm{hpw}$. (F) Total TNF promotor-driven fluorescent

543 area at the wound site at $24 \mathrm{hpw}$.

545 Supplementary Figure 1: Effects of SCFA administration on zebrafish hemostasis.

546 (A) Clotting at $2 \mathrm{hpw}$ following a tail wound in $5 \mathrm{dpf}$ zebrafish. (B) Thrombosis at $3 \mathrm{hpw}$

547 following a tail wound in 5 dpf zebrafish. 
bioRxiv preprint doi: https://doi.org/10.1101/2020.05.13.069997; this version posted May 15, 2020. The copyright holder for this preprint (which

was not certified by peer review) is the author/funder, who has granted bioRxiv a license to display the preprint in perpetuity. It is made available under aCC-BY-NC 4.0 International license.

\section{Supplementary Figure 2: Expression of hcar1 in germ-free embryos}

550 Quantification of hcarl expression in guts and bodies dissected from conventionally raised

551 and germ-free embryos. 


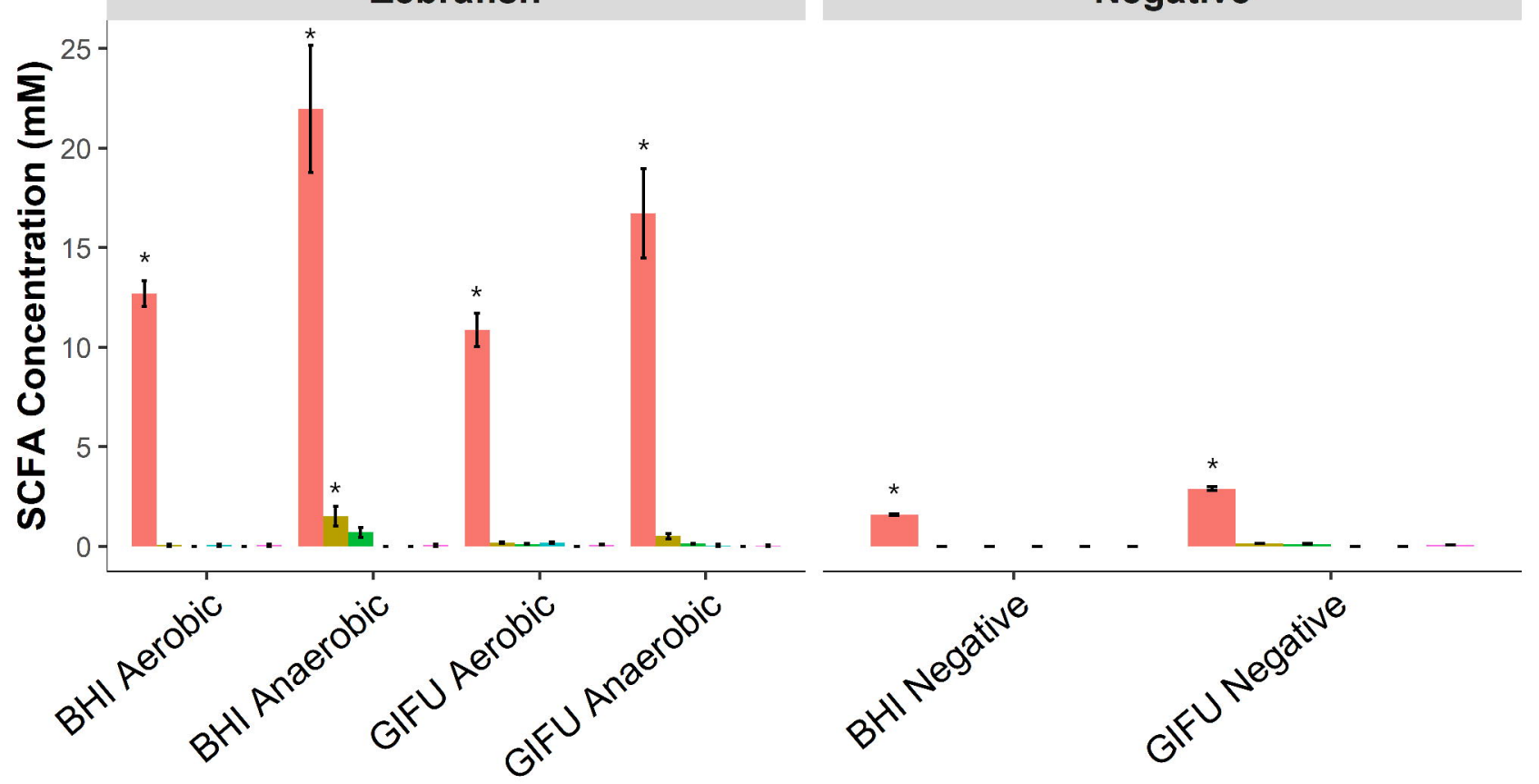

SCFA

Acetate

Propionate

Butyrate

Isobutyrate

Valerate

Isovalerate

\section{Conditions}




\section{Standard cut site}

Fin cut site for live imaging

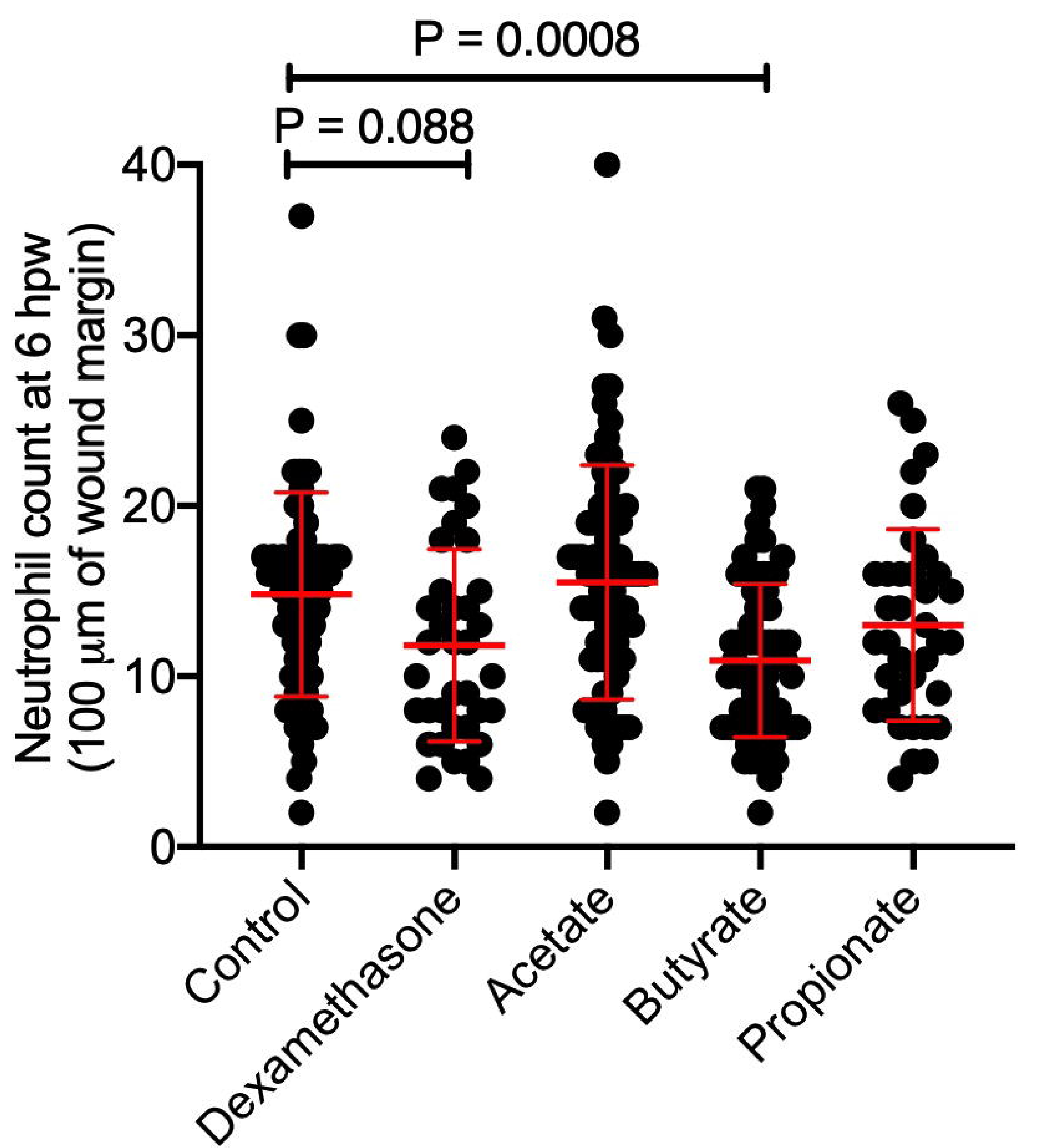

C $\quad P=0.0044 \quad D$
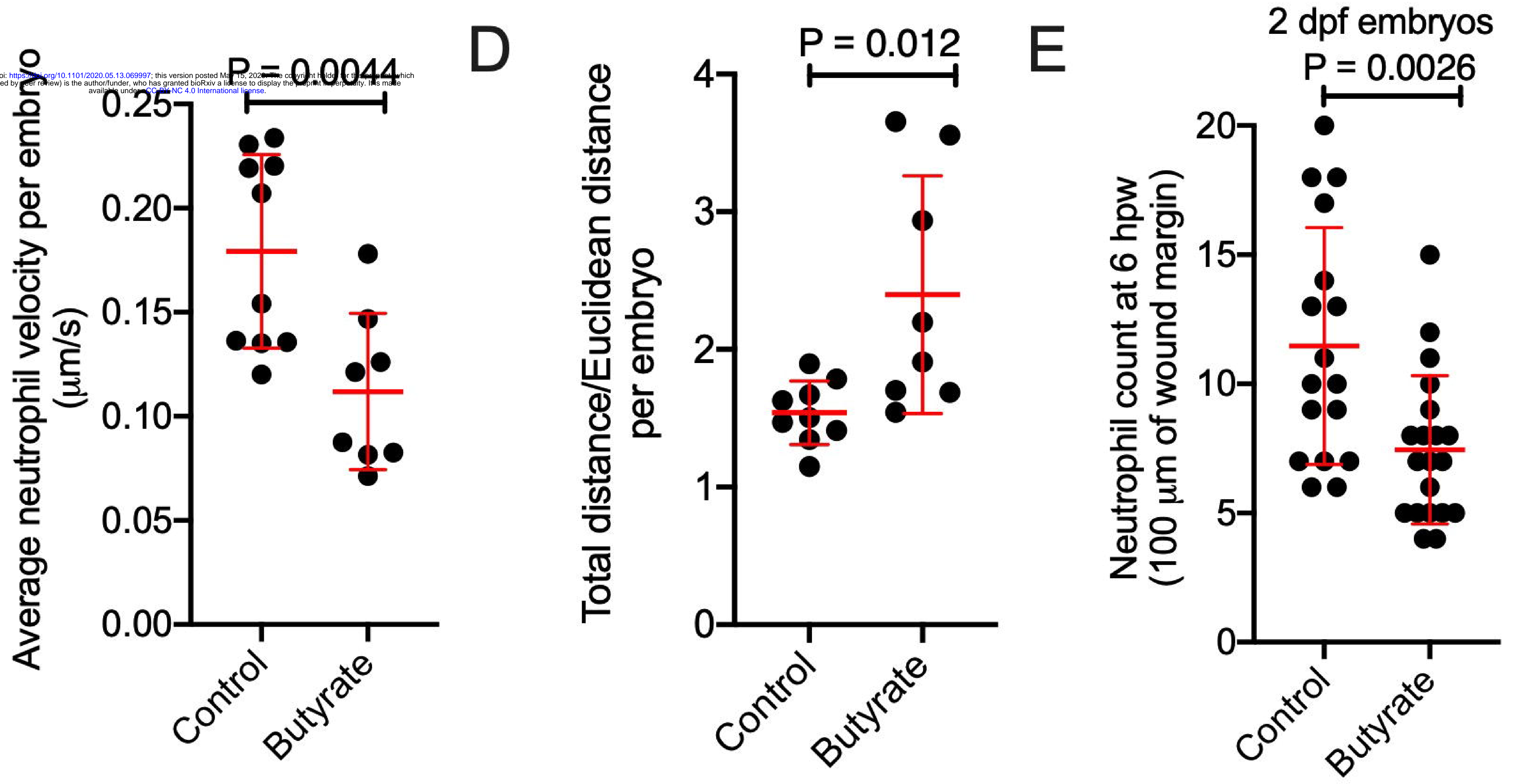


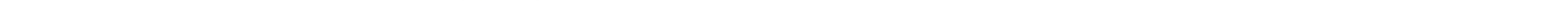




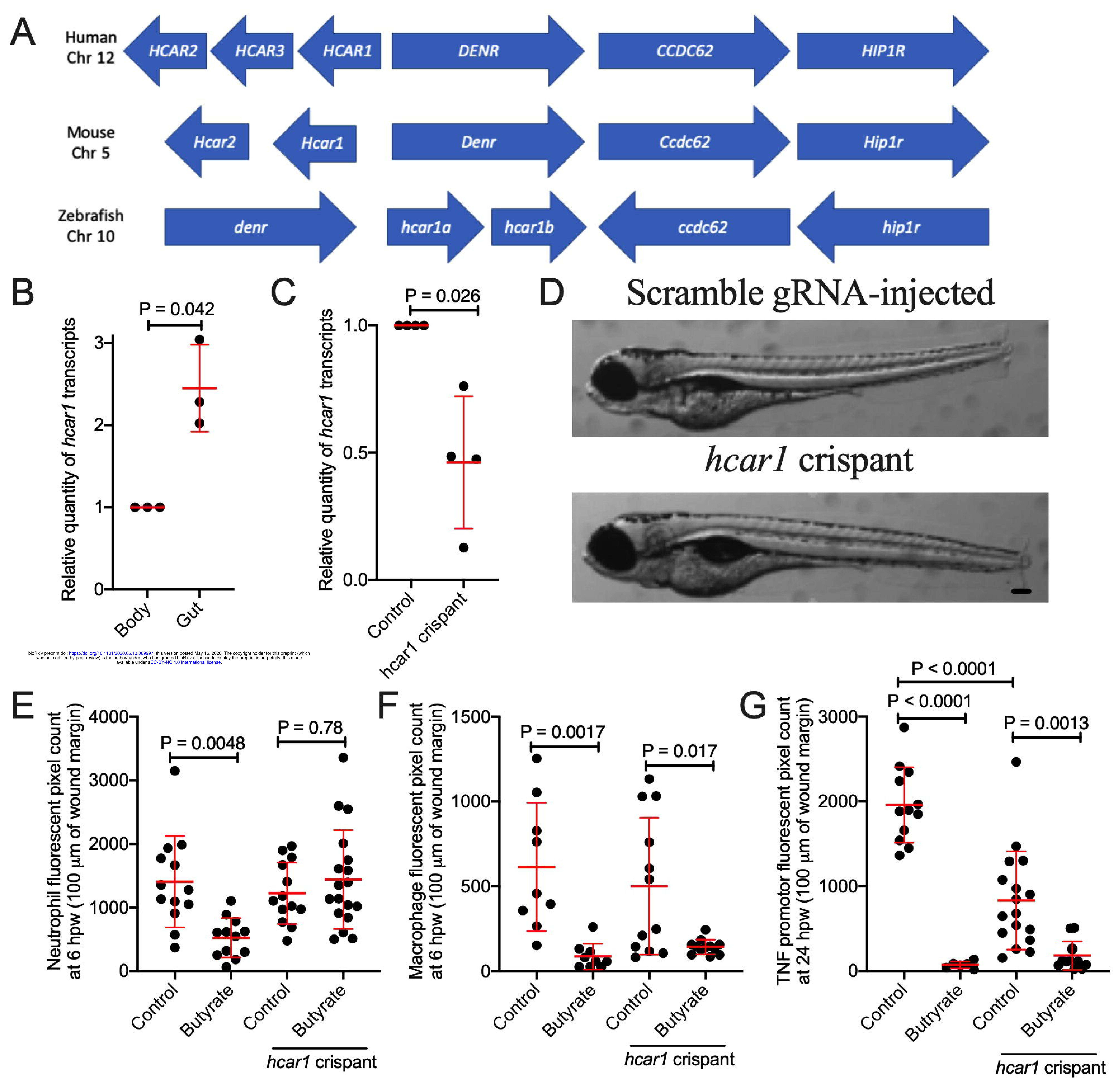

